Pacific Journal of Mathematics

MULTIPLICATIVITY-PRESERVING ARITHMETIC POWER 


\section{MULTIPLICATIVITY-PRESERVING ARITHMETIC POWER SERIES}

\section{DAVID REARICK}

In the Dirichlet algebra of arithmetic functions let the operator $A$ be represented by an arithmetic power series $A f=\Sigma a(F) f^{F}$. A condition on the coefficients $a(F)$ is derived which is necessary and sufficient for $A f$ to be multiplicative whenever $f$ is multiplicative.

1. Introduction. In [2] a factorization $F$ was defined to be a nonnegative integer-valued arithmetic function having $F(1)=0$ and $F(n) \neq 0$ for at most finitely $n$. The index of $F$ was defined by $i(F)=\prod_{j=1}^{\infty} j^{F(j)}$. If $f$ is any arithmetic function, we defined $f^{F}=$ $\prod_{j=1}^{\infty}[f(j)]^{F^{(j)}}$ with the understanding that $0^{0}=1$. If $a(F)$ is a mapping from factorizations into the real or complex numbers, we wrote

$$
A f=\sum a(F) f^{F}
$$

as an abbreviation for the arithmetic function $A f$ whose value on $n$ is equal to $\sum_{i(F)=n} a(F) f^{F}$. In [2] a series of the form (1) was called an arithmetic power series. Since for each $n$ the series is terminating, there is never any question of convergence. Such a series defines an operator $A$ on the Dirichlet algebra of arithmetic functions, and the theory of these operators has been investigated in [1] and [2].

In particular, if $r$ is a real number, the Dirichlet $r$ th power of an arithmetic function $f$ is represented, when $f(1)=1$, by an arithmetic power series $\Sigma\left(\begin{array}{l}r \\ F\end{array}\right) f^{F}$. The symbol $\left(\begin{array}{l}r \\ F\end{array}\right)$ was defined in [2]. It is known [1, Theorem 5] that $f^{r}$ is multiplicative whenever $f$ is, and therefore the series $\Sigma\left(\begin{array}{l}r \\ F\end{array}\right) f^{F}$ is an example of a multiplicativitypreserving arithmetic power series. The present paper is devoted to determining a necessary and sufficient condition on the coefficients $a(F)$ in order that the general series (1) preserve multiplicativity. The method, and the statement of the result (Theorem 1), depend on a certain equivalence relation between factorizations, to be introduced below.

\section{Equivalent factorizations.}

Definition 1 . If $F$ and $F^{\prime}$ are two factorizations, we say $F$ is 
equivalent to $F^{\prime}$, written $F \sim F^{\prime}$, if $f^{F}=f^{F^{\prime}}$ for every multiplicative arithmetic function $f$.

It is obvious that this is an equivalence relation. An example of a pair of nonequal but equivalent factorizations may be constructed by taking $F(2)=F(3)=F^{\prime}(6)=1$, with all other values being zero. Then $f^{F}=f(2) f(3)=f(6)=f^{F^{\prime}}$ for every multiplicative $f$. Two equivalent factorizations $F$ and $F^{\prime}$ necessarily have the same index, for if we choose the particular multiplicative function $f(n)=n$, we have $i(F)=f^{F}=f^{F^{\prime}}=i\left(F^{\prime}\right)$.

Definition 2. We shall use the letter $C$ to denote an equivalence class of factorizations. The index $i(C)$ of an equivalence class $C$ is defined to be the index of the factorizations $F$ belonging to $C$. If $f$ is multiplicative, we denote by $f^{C}$ the common value of $f^{F}$ for all $F \in C$. If $F_{1} \in C_{1}$ and $F_{2} \in C_{2}$, we define $C_{1}+C_{2}$ to be the equivalence class containing the factorization $F_{1}+F_{2}$.

It is obvious that the definition of $C_{1}+C_{2}$ is unambiguous.

If the operator (1) is applied to a multiplicative $f$, the sum over all factorizations $F$ of index $n$ reduces to a sum over all classes $C$ of index $n$, thus:

$$
A f(n)=\sum_{i ! T_{i}=n} a(F) f^{F}=\sum_{i(C,=n} f^{C} \sum_{F \in C} a(F)
$$

Therefore, insofar as its action on multiplicative functions is concerned, an arithmetic power series is determined by the sums of its coefficients over equivalence classes of factorizations, and it is natural to make the following definition:

Definition 3. $a^{*}(C)=\sum_{F \in C} a(F)$.

Thus, when $f$ is multiplicative, we may write

$$
A f(n)=\sum_{i(C)=n} a^{*}(C) f^{c}
$$

The main theorem may now be stated as follows.

Theorem 1. The arithmetic function $A f=\sum a(F) f^{F}$ is multiplicative whenever $f$ is, if and only if the following pair of conditions holds:

$$
a^{*}\left(C_{1}+C_{2}\right)=a^{*}\left(C_{1}\right) a^{*}\left(C_{2}\right)
$$


for every pair of equivalence classes $C_{1}$ and $C_{2}$ having relatively prime indices, and

$$
a^{*}(0)=1
$$

where 0 is the class containing the zero factorization.

3. Lemmas. Let those positive integers which are prime powers be arranged in increasing order. Let $x_{1}, x_{2}, \cdots$ be an arbitrary sequence of complex numbers. We may construct a multiplicative function $f$ by setting $f(1)=1$ and, it $p^{\nu}$ is the $k$ th prime power, defining

$$
f\left(p^{2}\right)=x_{k} .
$$

The requirement that $f$ be multiplicative then defines $f(n)$ for all positive integers $n$. Furthermore, every multiplicative $f$ arises from exactly one particular choice of the sequence $\left\{x_{k}\right\}$. (Following the usual convention, we do not consider the identically zero function to be multiplicative.)

These observations establish a one-to-one correspondence between the set of all multiplicative functions and the set of all sequences of variables $\left\{x_{k}\right\}$. Under this correspondence we may associate, with each factorization $F$, an expression $f^{F}$ which is a monomial (with coefficient 1 ) in certain of the variables $x_{k}$. We note that a given variable $x_{k}$ cannot appear in this monomial if it does not correspond, in (5), to a prime power divisor of $i(F)$, since, by definition of index $F(j)=0$ if $j$ does not divide $i(F)$.

Lemma 1. Two factorizations $F$ and $F^{\prime}$ are equivalent if and only if the two corresponding monomials $f^{F}$ and $f^{F^{\prime}}$ are identical.

Proof. It is familiar from algebra [3, Chapter 4] that if two polynomials always agree in value while each variable $x_{k}$ is assigned infinitely many different values, holding the others fixed, then the two polynomials are identical. The converse part of the assertion is trivial.

Lemma 1 shows that equivalence classes of factorizations may be identified with monomials in an arbitrary finite number of variables. Also, it is clear that each equivalence class of prime power index $p^{\nu}$ consists of a single factorization.

Lemma 2. Let $F_{1}, \cdots, F_{r}$ be nonequivalent factorizations. Suppose that, for every multiplicative $f$, the linear combination $\sum_{j=1}^{r} b_{j} f^{F_{j}}$ is equal to zero. Then each of the coefficients $b_{j}$ is zero. 
Proof. The linear combination referred to in the lemma is a polynomial in certain of the variables $x_{k}$, and the numbers $b_{j}$ are precisely its coefficients, since by Lemma 1 no two of the monomials $f^{F_{j}}$ are identical. As in the proof of Lemma 1, each of these coefficients must be zero.

Lemma 3. Let $F, F^{\prime}, G$, and $G^{\prime}$ be factorizations, with $i(F)=$ $i\left(F^{\prime}\right)=m$ and $i(G)=i\left(G^{\prime}\right)=n$, and assume $m$ and $n$ are relatively prime. Suppose $F+G \sim F^{\prime}+G^{\prime}$. Then $F \sim F^{\prime}$ and $G \sim G^{\prime}$.

Proof. As observed earlier, each variable $x_{k}$ appearing in the monomial $f^{F}$ corresponds, in (5), to a prime power divisor of $\mathrm{m}$. Similarly, $f^{G}$ contains only variables corresponding to prime power divisors of $n$. Since $(m, n)=1$, these two sets of variables are disjoint. Applying the same reasoning to $F^{\prime}$ and $G^{\prime}$, we see that no variable appearing in either $f^{F}$ or $f^{F^{\prime}}$ can appear in either $f^{G}$ or $f^{G^{\prime}}$, and conversely. By hypothesis we have $f^{F} f^{G}=f^{F+G}=f^{F^{\prime}+G^{\prime}}=$ $f^{F^{\prime}} f^{G^{\prime}}$ for all multiplicative $f$, or equivalently $f^{F} / f^{F^{\prime}}=f^{G^{\prime}} / f^{G}$. Since opposite sides of this identity are rational functions in disjoint sets of independent variables, both sides must be equal to a constant $B$. In the identity $f^{F}=B f^{F^{\prime}}$, putting $f(k)=1$ for all $k$, we obtain $B=$ 1. Therefore $f^{F}=f^{F^{\prime}}$ and $f^{G}=f^{G^{\prime}}$, meaning $F \sim F^{\prime}$ and $G \sim G^{\prime}$.

LEMmA 4. Let $F_{1}, \cdots, F_{r}$ be nonequivalent factorizations of index $m$. Let $G_{1}, \cdots, G_{s}$ be nonequivalent factorizations of index $n$. Assume $(m, n)=1$. Suppose that, for every multiplicative $f$, the linear combination $\sum_{j=1}^{r} \sum_{k=1}^{s} b_{j k} f^{F_{j}+G_{k}}$ is equal to zero. Then each of the coefficients $b_{j k}$ is zero.

Proof. By Lemma 3 the factorizations $F_{j}+G_{k}$ are all nonequivalent, and the result then follows from Lemma 2.

Lemma 5. Let $F$ be a factorization of index $m n$, where $(m, n)=1$. Then there exist factorizations $F_{1}$ and $F_{2}$, of indices $m$ and $n$ respectively, such that $F \sim F_{1}+F_{2}$. Furthermore, if $F_{1}^{\prime}$ and $F_{2}^{\prime}$ also satisfy these conditions, then $F_{1} \sim F_{1}^{\prime}$ and $F_{2} \sim F_{2}^{\prime}$. In other words, if $(m, n)=1$, then each equivalence class of index $m n$ is the sum of a unique pair of classes of indices $m$ and $n$ respectively.

Proof. The uniqueness part follows immediately from Lemma 3. As regards the existence of $F_{1}$ and $F_{2}$, we claim that the pair defined as follows will satisfy the requirements: 


$$
\begin{aligned}
F_{1}(k) & =0 & & \text { if } k=1 \\
& =\sum_{(j, m)=k} F(j) & & \text { if } k>1 \\
F_{2}(k) & =0 & & \text { if } k=1 \\
& =\sum_{(j, n)=k} F(j) & & \text { if } k>1 .
\end{aligned}
$$

To check this, choose any multiplicative $f$. Then

$$
\begin{aligned}
f^{F_{1}+F_{2}} & =f^{F_{1}} f^{F_{2}}=\prod_{k=1}^{\infty}[f(k)]^{F_{1}(k)} \prod_{k=1}^{\infty}[f(k)]^{F^{2}(k)} \\
& =\prod_{j=1}^{\infty}[f((j, m))]^{F(j)} \prod_{j=1}^{\infty}[f((j, n))]^{F^{(j)}} \\
& =\prod_{j=1}^{\infty}[f((j, m)) f((j, n))]^{F(j)} \\
& =\prod_{j=1}^{\infty}[f((j, m)(j, n))]^{F(j)} \\
& =\prod_{j=1}^{\infty}[f((j, m n))]^{F^{(j)}}=\prod_{j=1}^{\infty}[f(j)]^{F^{(j)}}=f^{F},
\end{aligned}
$$

where in the last step we use the fact that $F(j)=0$ if $j$ does not divide $m n$. Therefore $F \sim F_{1}+F_{2}$. To find the indices of $F_{1}$ and $F_{2}$, we first observe that $i\left(F_{1}\right) i\left(F_{2}\right)=i\left(F_{1}+F_{2}\right)=i(F)=m n$. Also, if we choose for $f$ the identity function $f(k)=k$, we have $i\left(F_{1}\right)=$ $f^{F_{1}}=\Pi_{j=1}^{\infty}(j, m)^{F(j)}$, and each factor in the product is relatively prime to $n$, so $i\left(F_{1}\right)$ is relatively prime to $n$. Similarly, $i\left(F_{2}\right)$ is relatively prime to $m$. Therefore $i\left(F_{1}\right)=m$ and $i\left(F_{2}\right)=n$.

4. Proof of Theorem 1. First assume conditions (3) and (4) hold. Choose any multiplicative $f$, and let $m$ and $n$ be relatively prime. We are to show that $A f(m n)=A f(m) A f(n)$ and $A f(1)=1$. By Lemma 5, each equivalence class $C$ of index $m n$ is the sum of a unique pair of classes $C_{1}+C_{2}$ where $i\left(C_{1}\right)=m$ and $i\left(C_{2}\right)=n$. Remembering (2), we may evaluate $A f(m n)$ as follows:

$$
\begin{aligned}
A f(m n) & =\sum_{i(C)=m n} a^{*}(C) f^{C}=\sum_{i\left(C_{1}\right)=m} \sum_{i\left(C_{2}\right)=n} a^{*}\left(C_{1}+C_{2}\right) f^{C_{1}+C_{2}} \\
& =\sum_{i\left(C_{1}\right)=m} a^{*}\left(C_{1}\right) f^{C_{1}} \sum_{i\left(C_{2}\right)=n} a^{*}\left(C_{2}\right) f^{C_{2}}=A f(m) A f(n) .
\end{aligned}
$$

Also, $A f(1)=a^{*}(0) f 0=1$.

To prove the converse, assume the operator $A$ preserves multiplicativity. Choose $m$ and $n$ relatively prime, and let $f$ be any multiplicative function. Proceeding as in the last computation above, we have

$$
\begin{aligned}
0 & =A f(m n)-A f(m) A f(n) \\
& =\sum_{i\left(C_{1}\right)=m} \sum_{i\left(C_{2}\right)=n} f^{C_{1}+C_{2}}\left[a^{*}\left(C_{1}+C_{2}\right)-a^{*}\left(C_{1}\right) a^{*}\left(C_{2}\right)\right] .
\end{aligned}
$$


This double sum is a linear combination of the type considered in Lemma 4, and therefore, by the result of that lemma, the expression in square brackets is equal to zero for all $C_{1}$ and $C_{2}$ in the sum. That is, equation (3) is satisfied. Also, (4) is satisfied because $1=$ $A f(1)=a^{*}(0) f 0=a^{*}(0)$. This completes the proof of Theorem 1 .

5. Further consequences. We wish to show how to construct all solutions $a^{*}(C)$ of (3) which also satisfy (4) (and which we shall refer to as nontrivial solutions of (3)). Given a nontrivial solution $a^{*}(C)$ of (3), we can recover (nonuniquely) by Definition 3 the coefficients $a(F)$ of an arithmetic power series (1) which preserves multiplicativity, and the class of such series will then be completely characterized.

Lemma 6. Let $C$ be an equivalence class whose index is greater than 1 and has prime factorization $i(C)=p_{1}^{\nu_{1}}, \cdots, p_{r}^{\nu}$. Then there are unique classes $C_{1}, \cdots, C_{r}$, of indices $p_{1}^{\nu_{1}}, \cdots, p_{r}^{\nu r}$ respectively, such that $C=C_{1}+\cdots+C_{r}$.

Proof. Apply Lemma 5 repeatedly to the $r$ maximal prime power divisors $p_{1}^{\nu_{1}}, \cdots, p_{r}^{\nu}$ of $i(C)$.

Lemma 7. $a^{*}(C)$ is a nontrivial solution of (3) if and only if $a^{*}(0)=1$ and

$$
a^{*}(C)=\prod_{k=1}^{r} a^{*}\left(C_{k}\right)
$$

whenever $i(C)>1$, where the classes $C_{1}, \cdots, C_{r}$ are related to $C$ as in Lemma 6.

Proof. Equation (6) is obtained from (3) by applying the latter repeatedly to the maximal prime power divisors of $i(C)$. Conversely, (3) is obtained from (6) by applying (6) to the prime decomposition of $m n$, separating the maximal prime power divisors of $m$ from those of $n$.

Lemma 7 gives us a process for constructing all nontrivial solutions of (3). The method is analogous to that used at the beginning of $\S 3$ to construct all multiplicative functions, namely:

THEOREM 2. The nontrivial solutions $a^{*}(C)$ of (3) are exactly those which take the value 1 on the zero class and are defined arbitrarily on classes of prime power index, the definition then being extended to all $C$ by the product formula (6). 
Finally, we shall determine the number of equivalence classes of index $n$. Let this number be denoted by $E(n)$. It follows from Lemma 5 that $E(n)$, as an arithmetic function, is multiplicative. Therefore, it suffices to evaluate this function on prime powers $p^{2}$. Since each class of index $p^{\nu}$ contains only one factorization, $E\left(p^{\nu}\right)$ is equal to the number of factorizations of index $p^{\nu}$, and this is evidently just the number of unrestricted partitions of $\nu$. These observations yield the following explicit formula for $E(n)$ :

THEOREM 3.

$$
\begin{aligned}
& E(1)=1 \\
& E(n)=\prod_{p^{\nu / n}} p(\nu) \quad \text { if } n>1,
\end{aligned}
$$

where $p(\nu)$ is the partition function, and the product is extended over all maximal prime power divisors $p^{2}$ of $n$.

\section{REFERENCES}

1. D. Rearick, Operators on algebras of arithmetic functions, Duke Math J., 35 (1968), 761-766.

2. - The trigonometry of numbers, Duke Math. J., 35 (1968), 767-776.

3. B. L. Van der Waerden, Modern Algebra, Vol. I, 2nd ed., New York, 1953.

Received March 20, 1973.

UNIVERSITY OF COLORADO 



\section{PACIFIC JOURNAL OF MATHEMATICS}

EDITORS

RICHARD ARENS (Managing Editor)

University of California

Los Angeles, Calıfornia 90024

R. A. Beaumont

University of Washington

Seattle, Washington 98105
J. DugundJI

Department of Mathematics

University of Southern California

Los Angeles, California 90007

D. Gilbarg and J. Milgram

Stanford University

Stanford, California 94305

\section{ASSOCIATE EDITORS}
E. F. BECKENBACH
B. H. NeumanN
F. WOLF
K. YOSHIDA

\section{SUPPORTING INSTITUTIONS}

UNIVERSITY OF BRITISH COLUMBIA

CALIFORNIA INSTITUTE OF TECHNOLOGY

UNIVERSITY OF CALIFORNIA

MONTANA STATE UNIVERSITY

UNIVERSITY OF NEVADA

NEW MEXICO STATE UNIVERSITY

OREGON STATE UNIVERSITY

UNIVERSITY OF OREGON

OSAKA UNIVERSITY
UNIVERSITY OF SOUTHERN CALIFORNIA

STANFORD UNIVERSITY

UNIVERSITY OF TOKYO

UNIVERSITY OF UTAH

WASHINGTON STATE UNIVERSITY

UNIVERSITY OF WASHINGTON

AMERICAN MATHEMATICAL SOCIETY NAVAL WEAPONS CENTER 


\section{Pacific Journal of Mathematics}

\section{Vol. 55, No. $1 \quad$ September, 1974}

Robert Lee Anderson, Continuous spectra of a singular symmetric

differential operator on a Hilbert space of vector-valued functions . . . $\quad 1$

Michael James Cambern, The isometries of $L^{p}(X, K) \ldots \ldots \ldots \ldots \ldots . . \ldots$

R. H. Cameron and David Arne Storvick, Two related integrals over spaces of continuous functions ................................

Gary Theodore Chartrand and Albert David Polimeni, Ramsey theory and

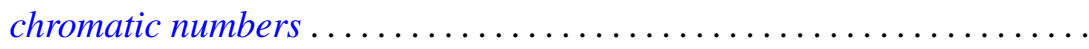

John Deryck De Pree and Harry Scott Klein, Characterization of collectively compact sets of linear operators ...................

John Deryck De Pree and Harry Scott Klein, Semi-groups and collectively compact sets of linear operators ....................... 55

George Epstein and Alfred Horn, Chain based lattices.............. 65

Paul Erdős and Ernst Gabor Straus, On the irrationality of certain series . . 85

Zdeněk Frolík, Measurable uniform spaces................... 93

Stephen Michael Gagola, Jr., Characters fully ramified over a normal

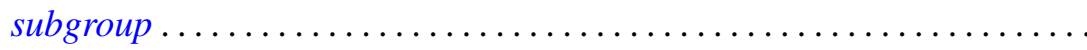

Frank Larkin Gilfeather, Operator valued roots of abelian analytic

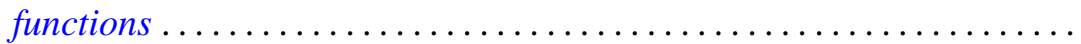

D. S. Goel, A. S. B. Holland, Cyril Nasim and B. N. Sahney, Best approximation by a saturation class of polynomial operators

James Secord Howland, Puiseux series for resonances at an embedded

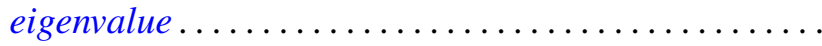

David Jacobson, Linear GCD equations .................

P. H. Karvellas, A note on compact semirings which are multiplicative

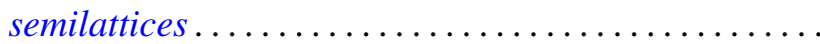

Allan Morton Krall, Stieltjes differential-boundary operators. II . .

D. G. Larman, On the inner aperture and intersections of convex sets

S. N. Mukhopadhyay, On the regularity of the $P^{n}$-integral and its application to summable trigonometric series ....... .

Dwight Webster Read, On $(J, M, m)$-extensions of Boolean algebras ....

David Francis Rearick, Multiplicativity-preserving arithmetic power series.

Indranand Sinha, Characteristic ideals in group algebras

Charles Thomas Tucker, II, Homomorphisms of Riesz spaces . . .

Kunio Yamagata, The exchange property and direct sums of indecomposable injective modules. 